http://dx.doi.org/10.35381/r.k.v6i2.1234

\title{
Efecto de la actividad física en personas con epilepsia. Un análisis documental y bibliográfico
}

\section{Effect of physical activity in people with epilepsy. Documentary and} bibliographic análisis

Sarita Emilia Delgado-Salgado

sarita.delgado@est.ucacue.edu.ec

Universidad Católica de Cuenca, Azogues

Ecuador

https://orcid.org/0000-0002-7223-475X

Zoila Guillermina Torres-Palchisaca

ztorresp@ucacue.edu.ec

Universidad Católica de Cuenca, Azogues

Ecuador

https://orcid.org/0000-0003-3078-6465

Recepción: 25 de enero 2021

Revisado: 20 de febrero 2021

Aprobación: 30 de abril 2021

Publicación: 15 de mayo 2021 


\title{
RESUMEN
}

En el siguiente artículo de revisión bibliográfica, se discuten los efectos beneficiosos que tiene la actividad física para las personas con epilepsia. Para algunos autores de las investigaciones estudiadas; el beneficio de estas actividades físicas puede ser muy positivo frente a las contraindicaciones para practicarlo. Es así que la investigación realiza un estudio sobre el efecto de la misma en la epilepsia siendo esta un trastorno cerebral que se caracteriza por la aparición de crisis epilépticas. Todo esto permitirá evitar la desinformación y toma de decisiones equivocadas en toda la sociedad y más aún el circulo social que gira alrededor del paciente. Además, se infiere que la actividad física es un punto fundamental para mantener el cuerpo y mente activos; la misma que no permite que otras enfermedades desestabilicen o ataquen al paciente.

Descriptores: Enfermedad del sistema nervioso; deporte; educación física. (Palabras tomadas del Tesauro UNESCO).

\begin{abstract}
In the following literature review article, the beneficial effects of physical activity for people with epilepsy are discussed. For some authors of the studied investigations; the benefit of these physical activities can be very positive in front of the contraindications to practice it. This is how the research carries out a study on the effect of the same in epilepsy, this being a brain disorder characterized by the appearance of epileptic seizures. All this will allow avoiding misinformation and wrong decisions in the whole society and even more in the social circle that revolves around the patient. In addition, it is inferred that physical activity is a fundamental point to keep the body and mind active; the same that does not allow other diseases to destabilize or attack the patient.
\end{abstract}

Descriptors: Nervous system diseases; sport; physical education. (Words taken from the UNESCO Thesaurus). 


\section{INTRODUCCIÓN}

La organización mundial de la salud nos habla de la actividad física como todos los movimientos realizados por el sistema muscular y que requieren de un gasto energético. Esta actividad se refiere a todo el movimiento incluso los que son realizados en momentos de recreación o en la parte laboral. No podemos olvidar que esta actividad mejora la salud (Organziación mundial de la salud, OMS, 2020).

Por otro lado, históricamente se habla sobre la epilepsia y se informa que esta es una enfermedad neurológica crónica, según estudios se habla que la misma afecta a un porcentaje de personas del mundo entero, en un promedio de 50 millones de personas. Se caracteriza por convulsiones recurrentes, que son episodios transitorios de movimiento estos son los que consiguen involucrar una parte del cuerpo (parcial) o todo el cuerpo (generalizado) y en instantes se escoltan de quebranto de conciencia (OMS, 2019).

A pesar de que en la sociedad actual se hace hincapié en el ejercicio y el buen estado físico, los pacientes epilépticos suelen quedar excluidos de la participación en la actividad física. Esta renuencia a permitir que los pacientes con epilepsia participen en el deporte proviene tanto de los médicos como de los padres sobreprotectores e incluso de la persona que lo padece, ya sea por el temor a que el ejercicio pueda empeorar la condición del paciente, predisponer lesiones o que la fatiga resultante pueda precipitar nuevas convulsiones. Es así que durante muchos años se ha debatido si los pacientes con epilepsia pueden participar en programas de acondicionamiento físico, deportes recreativos o competitivos, en particular de colisión o contacto (Acebes, 2018).

Es así que la actividad o ejercicio físico va tomando importancia en personas epilépticas en el proceso del tiempo; conforme los estudios médicos especialmente los neurológicos van avanzando la actividad física es recomendada para pacientes epilépticos, siempre y cuando sea supervisado por profesionales en el área y no sea descuidada en ningún momento (Dustin, 2019). 
De acuerdo, al objetivo de este estudio podemos decir que es comparar las ventajas y desventajas propuestas por los investigadores en relación a la actividad física en individuos con epilepsia de acuerdo al análisis de información científica ya existente.

\section{DESARROLLO DE LA REVISIÓN}

El presente trabajo fue elaborado con el método de investigación o revisión bibliográfica sistemática de carácter descriptivo - exploratorio; desarrollando así una revisión del tema conjuntamente con su problemática y todos los demás componentes durante todo este tiempo (Parra-Zhizhingo, et al. 2020). Dicho análisis documental se efectuó investigando fuentes primarias como son artículos científicos de alto impacto en repositorios internacionales como Google schoolar, revistas internacionales, tesis de grado, libros, informes y demás relacionados con el tema, de donde se extrajo conceptos o definiciones respecto a la actividad física y epilepsia su relación médica, física, psicológica y los efectos a evidenciar en la vida cotidiana del paciente 0 deportista con epilepsia. Además, se trató de definir los conceptos más relevantes que relacionan la actividad física o deporte con la epilepsia (Puma-Pañora, et al. 2020). Es importante reconocer que en cada investigación encontrada fue realizada en otros países, la información relevante como aquellas no se encuentra en nuestro país y esto no permite avance para las personas que lo padecen por el mismo hecho de falta de información (Erazo-Álvarez \& Narváez-Zurita, 2020).

\section{Limitación bibliográfica}

En la búsqueda bibliográfica se registró las siguientes categorías: informes, artículos científicos, artículos médicos, ensayos clínicos controlados, ensayos clínicos no controlados, informes de casos y encuestas. Los juicios de eliminación fueron los resúmenes de disertaciones por la falta de detalles sobre la metodología. La búsqueda se limitó a artículos en inglés, portugués y español. De 554 artículos identificados, 334 fueron rechazados después de leer el título y el resumen porque no se consideraban relevantes para la temática y 198 artículos más fueron separados por la misma razón 
tras la exploración del texto completo. Los 22 artículos restantes se contuvieron en este articulo bibliográfico.

Después de una introducción a la temática del presente artículo es importante mencionar que el lector debe conocer a fondo las definiciones, clasificaciones, causas, síntomas, repercusiones, desarrollo de actividades, etc.; para así poder entender el porqué del estudio, es así que a continuación se plantea lo anteriormente dicho.

\section{La epilepsia}

Es un trastorno cerebral que se define por una tendencia continua a la aparición de crisis epilépticas, el mismo que posee efectos neurobiológicas, cognitivas, psicológicas y sociales de esta enfermedad. El diagnostico de epilepsia necesita de más de una crisis epiléptica (Fisher, et al. 2014). Podemos decir que las convulsiones se deben a descargas eléctricas en el cerebro y por ende en sus células específicas como son las neuronas, las mismas que se encuentran en todo en nuestro cerebro (OMS, 2020). Debemos tomar en cuenta que los episodios son movimientos rápidos e inconscientes que pueden perturbar por medio de: convulsiones parciales o convulsiones generalizadas. Un dato importante sobre las crisis es que custodian de pérdida de la consciencia y del control de los esfínteres (Carrizosa-Moog, 2017). Aquellos entes con esta enfermedad neurológica suelen sufrir más dificultades físicas en algunos casos fracturas, hematomas y en otros casos derivados de contusiones relacionados con las mismas crisis. Existen tasas de perturbaciones psicosociales, entre ellos ansiedad y depresión. Por ende, el riesgo de muerte prematura es mucho mayor en un paciente con epilepsia frente a la de una persona que no padezca esta enfermedad. Los estudios hablan que estos índices se encuentran más altos en la población con ingresos medios y bajos (OMS, 2020). 


\section{Clasificación de las convulsiones}

a) Las generalizadas perjudican a los 2 lados del cerebro.

b) Las de ausencia, pueden originar un parpadeo rápido o la mirada fija a lo lejos por pocos segundos.

c) Las tonicoclónicas, también llamadas epilepsia mayor o gran mal, pueden hacer que la persona: grite, pierda el conocimiento, tenga rigidez o espasmos musculares. (Centro para el control de enfermedades, 2018).

\section{Causas y tratamiento}

La epilepsia no es contagiosa. De todos modos, existe varios mecanismos de padecimiento subyacentes pueden causar epilepsia, se desconoce el origen de la enfermedad cerca del $50 \%$ de los casos de todo el mundo (OMS, 2020). Las causas de la epilepsia se fraccionan en las clases siguientes: estructurales, genéticas, infecciosas, metabólicas, inmunológicas y desconocidas (Arida, et al. 2008). Al mismo por medio de un seguimiento clínico se puede llevar un control favorable frente a las convulsiones.

Con un procedimiento anticonvulsivante apropiado, hasta un $70 \%$ de los pacientes podrían vivir sin problema alguno. Estudios indican la opción de concluir con el tratamiento después de 2 años que no existan crisis; para ello, los factores clínicos son muy importantes, además de los sociales y personales que no son olvidados. Una etiología documentada de la crisis epiléptica y un patrón electroencefalográfico anormal son los dos factores predictivos más consistentes de la recurrencia de convulsiones (OMS, 2020).

\section{Repercusiones sociales y económicas}

La discriminación y la estigmatización social que envuelven a la enfermedad en si tienen mayor dificultad para vencer opiniones psicosociales antes que las propias convulsiones. Los entes epilépticos pueden ser centro de acoso o burla. La estigmatización del malestar puede hacer que los involucrados no busquen tratamiento para impedir mejorar su estado de salud (Escudero, 2007). 
La legislación basada en los derechos humanos puede impedir la discriminación y el quebrantamiento de los mismos, uno de los principales objetivos es de optimizar un servicio de salud que se merecen este grupo específico de personas, además incrementar la calidad de vida de las mismas (OMS, 2019).

\section{Actividad física y sus acompañantes}

Para fines de contextualización del tema de investigación, es necesario precisar conceptos relacionados con la actividad física y deporte:

Actividad física: son los movimientos que crean un consumo calórico, pero no necesariamente contiene o es parte de una planificación estructurada (Carpio, 2018). Ejercicio físico: Actividad física constituida, proyectada y repetitiva de movimientos, realizados para mejorar el estado físico de quien lo realiza (Carpio, 2018).

Deporte: es la actividad física que envuelve reglas o normas a desempeñar dentro de un determinado lugar como puede ser el campo de juego, cancha, pista u otros lugares siempre de la mano con la competitividad deportiva. Se encuentra institucionalizado es decir contiene federaciones, clubes, ligas barriales; esta necesita de competición con uno mismo o con los demás. Los deportes requieren capacidad física para su participación, pero existen otros que tienen mayor degaste de intelecto frente al físico, como es el caso del ajedrez (Ibarra-Angulo, 2018).

Estado físico o condición física: Atributos físicos y fisiológicos que las personas tienen o adquieren y que se relacionan con la habilidad para desarrollar actividad física, ejercicio y deporte en forma eficaz, eficiente, segura y saludable (Caamaño-Navarrete, et al. 2016).

Con relación al objetivo de investigación, el cual es conocer el impacto de la actividad física en las personas con epilepsia, realiza un enfoque en investigaciones más relevantes y recientes, incluyendo avances del circulo medico que dan soporte a la investigación en el caso de personas con esta condición y que realizan algún tipo de actividad física o deportiva. Así el artículo científico de (Capovilla, et al. 2016), mencionan que a menudo se desaconseja a las personas con epilepsia la práctica de deportes y ejercicio, sobre todo por miedo, sobreprotección y desconocimiento de los 
beneficios y los riesgos asociados a estas actividades. La evidencia útil propone que el ejercicio físico y la intervención activa en deportes pueden conmover favorablemente al control de las convulsiones, asimismo de originar beneficios psicosociales y de salud más extensos (Capovilla, et al. 2016).

El estudio de Volpato y colaboradores en el año 2017 indican que, la epilepsia es más que convulsiones e incluye un alto riesgo de comorbilidades y trastornos psicológicos, lo que sobrelleva una mala aptitud de vida, al respecto se puede completar esta idea manifestando que un estilo de vida sedentario en las personas con epilepsia, podrían contribuir a una peor salud y a problemas psicológicos (Volpato, et al. 2017).

En el mismo sentido se conoce que al momento de realizar actividad física o deporte se liberan endorfinas que realizan efectos positivos inclusive hasta horas posteriores. Se ocasionan instrumentos a nivel celular y molecular del sistema nervioso central, permitiendo que existan líneas más eficientes y vertiginosas entre diferentes sitios cerebrales como son lóbulos frontales, parietales o en si áreas generalizadas que son las más comunes (Andreu, 2016). De hecho, evidencia científica indica que el ejercicio y el deporte deben fomentarse para todas las personas con epilepsia, incluidos los niños y los adolescentes. Estudios anteriores han demostrado los efectos positivos del ejercicio como la baja de la frecuencia de las crisis, la mejora del estado cardiovascular y la ampliación de la calidad de vida, la autoestima y el bienestar (Vancini, et al. 2017). Los pacientes epilépticos, con unas previsiones apropiadas, pueden realizar varios deportes (Escudero M. , 2017), y empezar una nueva actividad deportiva es necesario varios puntos especiales como es el tipo de epilepsia, progreso y tiempo acontecido desde la última crisis, pues un paciente que no tiene control y posee crisis continuas puede tener riesgos grandes de caídas en las distintas actividades, es por ello que la práctica deportiva debe contener estrategias conjuntamente con el paciente (Escudero, 2007). También es muy necesario determinar qué tipo de medicación usa el deportista con esta afección y los efectos secundarios que se puedan presentar.

Libremente del deporte que se escoja efectuar se tratara de llegar al agotamiento extremo, las horas de sueño son sumamente importantes, la hidratación correcta, sin ninguna molestia dos horas antes de empezar a realizar el ejercicio (Escudero, 2007). 
Los pacientes tienen completamente contraindicados ciertos deportes como son: el boxeo, kickboxing, el buceo con bombona, el paracaidismo, el puénting y el alpinismo en solitario, los deportes de contacto y el tiro olímpico. Estos deportes mantienen un riesgo de desplome, además los de contacto y los motorizados, diseñan mucho riesgo para los deportistas y por lo tanto serán ejecutados bajo responsabilidad de quien lo practique (Escudero, 2017).

Si hablamos de los deportes más adecuados para un paciente epiléptico, podemos observar que son varios, aunque no podemos dejar de lado el riesgo así este sea mínimo.

\section{Tabla 1.}

Deportes recomendados para pacientes epilépticos.

\begin{tabular}{|l|}
\hline $\begin{array}{l}\text { Deportes acuáticos: } \\
\text { Natación: Informar al entrenador, compañeros y socorrista. En crisis } \\
\text { controladas. }\end{array}$ \\
\hline $\begin{array}{l}\text { Esquí Alpino: En el caso de realizarlo, usar casco. (Mejor realizar esquí de } \\
\text { fondo o travesía) }\end{array}$ \\
\hline Escalada con sujeción: Utilizar siempre casco. \\
\hline Gimnasia deportiva: Ejercicios de suelo y salto de potro permitidos. \\
\hline $\begin{array}{l}\text { Patinaje y ciclismo: Usar siempre casco y por zonas con poco trafico (pacientes } \\
\text { con crisis controladas) }\end{array}$ \\
\hline $\begin{array}{l}\text { Navegación, Kayak: Se puede realizar con crisis controladas, con canoas } \\
\text { antivuelco y chalecos salvavidas. }\end{array}$ \\
\hline Pesca: Realizar acompañado. \\
\hline
\end{tabular}

Fuente: Sánchez (2014). 
A pesar de todas las limitaciones que la epilepsia puede presentar, resulta muy alentador conocer que a nivel mundial han existido grandes deportistas que a pesar de su condición han demostrado que es posible realizar deporte incluso llegando al alto rendimiento, como es el caso del futbolista de la selección brasileña Ronaldo Nazario, o la atleta campeona olímpica de velocidad estadounidense, Florence Griffith Joyner. Por lo tanto, el padecer una enfermedad como la epilepsia no es un limitante para hacer actividad física o algún tipo de deporte en particular, siempre y cuando se consideren los debidos cuidados y precauciones (Bender-del-Busto \& HernándezToledo, 2017).

\section{APORTES GENERADOS DE LA REVISIÓN}

Una vez realizado el análisis de diferentes fuentes bibliográficas sobre nuestra investigación en personas con epilepsia se ha llegado a lo siguiente:

La actividad física o deporte es totalmente recomendada a pacientes que padecen epilepsia. No obstante, el ejercicio debería originarse después de hacerse una evaluación clínica cuidadosa, dentro de ella se considerará las ultimas convulsiones, posibles elementos precipitantes y el tipo de deporte (Cartagena, et al. 2020).

Se debe considerar el tipo de actividad o deporte para que el paciente pueda desenvolverse de la mejor manera en el ámbito y así no generar problemas médicos, psicológicos o sociales frente al entorno en donde se desplegará (Moreno, 2009).

Por otro lado, el incremento del tiempo que se dedica a la actividad física o deporte, no empeora la salud o diagnóstico médico más bien es una ayuda y mejora otros ámbitos que intervienen medicamente como son: aspectos cardiovasculares, aspectos hormonales, aspectos psicosociales, aspectos sedentarios, etc. No olvidemos que el diagnóstico médico es el principal punto de partida que permitirá a los demás profesionales y amateur realizar un buen trabajo conjuntamente con el paciente (Macas-Macas, et al. 2020).

Para culminar se sugiere realizar nuevas investigaciones aplicadas dentro de nuestro contexto en particular, para determinar el impacto que a largo plazo ejerce la práctica 
deportiva, en personas que desean practicar actividad física o cualquier tipo de deporte bajo las condiciones particulares de la epilepsia.

\section{FINANCIAMIENTO}

No monetario.

\section{AGRADECIMIENTO}

A la Universidad Católica de Cuenca y la Jefatura de Posgrados por apoyar el desarrollo de la investigación.

\section{REFERENCIAS CONSULTADAS}

Acebes, J. (2018). Revisión sistemática de epilepsia, actividad física, deporte y ejercicio [Systematic review of epilepsy, physical activity, sport and exercise]. Recuperado de https://n9.cl/v3x8q

Andreu, J. M. (2016). La Práctica de Actividad Física y su Relación con el Rendimiento Académico [The Practice of Physical Activity and its Relation to Academic Achievement]. El journal Revista de Educación Física, 34(4), 1-9. https://n9.cl/zbhto

Arida, R. M., Cavalheiro, E. A., da Silva, A. C., \& Scorza, F. A. (2008). Physical activity and epilepsy: proven and predicted benefits. Sports medicine (Auckland, N.Z.), 38(7), 607-615. https://doi.org/10.2165/00007256-200838070-00006

Bender-del-Busto, J., \& Hernández-Toledo, L. (2017). Consideraciones en el tratamiento del paciente con epilepsia. Artículo de revisión [Considerations in the treatment of the patient with epilepsy. A review article]. Revista Habanera De Ciencias MéDicas, 16(6), 912-926.

Caamaño-Navarrete, F., Delgado Floody, P., Jerez Mayorga, D., \& Osorio Poblete, A. (2016). Bajos niveles de rendimiento físico, VO2 MAX y elevada prevalencia de obesidad en escolares de 9 a 14 años de edad [Low levels of physical performance, VO2 MAX and high prevalence of obesity in schoolchildren aged 9 to 14 years]. Nutricion hospitalaria, 33(5), 565. https://doi.org/10.20960/nh.565 
Capovilla, G., Kaufman, K. R., Perucca, E., Moshé, S. L., \& Arida, R. M. (2016). Epilepsy, seizures, physical exercise, and sports: A report from the ILAE Task Force on Sports and Epilepsy. Epilepsia, 57(1), 6-12. https://doi.org/10.1111/epi.13261

Carpio, O. A. (2018). Nuevos planteamientos en la prescripción del ejercicio físico [New approaches to physical exercise prescription]. XIV Congreso peruano de nutrición (p. 24). Lima: Facultad de Medicina Humana.

Carrizosa-Moog, J. (2017). Epilepsia y actividad física: conceptos desde la perspectiva de las ciencias básicas. latreia, 30(3), 287-296. https://doi.org/10.17533/udea.iatreia.v30n3a04

Cartagena, Y., Cardona-Gallón, D. C., Isaza, S. P., \& Ladino, L. D. (2020). El ejercicio como estrategia terapéutica en la epilepsia: revisión bibliográfica [Exercise as a therapeutic strategy in epilepsy: a literature review]. Revista de neurologia, 71(1), 31-37. https://doi.org/10.33588/rn.7101.2020028

Centro para el control de enfermedades. (2018). Convulsiones [Seizures]. Recuperado de https://n9.cl/n9s5w

Dustin, I. H., Resnick, B., Galik, E., Klinedinst, N. J., Michael, K., Wiggs, E., \& Theodore, W. H. (2019). The Feasibility and Impact of the EMOVE Intervention on Self-efficacy and Outcome Expectations for Exercise in Epilepsy. The Journal of neuroscience nursing : journal of the American Association of Neuroscience Nurses, 51(2), https://doi.org/10.1097/JNN.0000000000000425

Erazo-Álvarez, J., \& Narváez-Zurita, C. (2020). Medición y gestión del capital intelectual en la industria del cuero - calzado en Ecuador [Measurement and management of intellectual capital in the leather industry - footwear in Ecuador]. Revista Arbitrada Interdisciplinaria Koinonía, 5(9), 437-467. http://dx.doi.org/10.35381/r.k.v5i9.662

Escudero, M. (2007). El ejercicio físico en la epilepsia [Physical exercise in epilepsy]. Medicina de familia-Semergen, 33(3), 127-139. https://doi.org/10.1016/S11383593(07)73863-7

Fisher, R. S., Acevedo, C., Arzimanoglou, A., Bogacz, A., Cross, J. H., Elger, C. E., Engel, J., Jr, Forsgren, L., French, J. A., Glynn, M., Hesdorffer, D. C., Lee, B. I., Mathern, G. W., Moshé, S. L., Perucca, E., Scheffer, I. E., Tomson, T., Watanabe, M., \& Wiebe, S. (2014). ILAE official report: a practical clinical definition of epilepsy. Epilepsia, 55(4),

475-482. https://doi.org/10.1111/epi.12550 
Ibarra-Angulo, C. E. (2015). El deporte [The sport]. Vida Científica Boletín Científico De La Escuela Preparatoria 4, 3(5).

Macas-Macas, A., García-Herrera, D., Álvarez-Lozano, M., \& Erazo-Álvarez, J. (2020). Dino Tim y el desarrollo lógico matemático en niños de Educación Inicial [Dino Tim and mathematical logical development in Early Childhood Education children]. Revista Arbitrada Interdisciplinaria Koinonía, 5(5), 281-299. http://dx.doi.org/10.35381/r.k.v5i5.1044

Moreno, A. (2009). Innovacion y experiencias educativas [Innovation and educational experiences]. Recuperado de https://n9.cl/mp2tr

OMS. (2019). Epilepsia [Epilepsy]. Recuperado de https://n9.cl/2m5v

OMS. (2020). Actividad física [physical activity]. Recuperado de https://n9.cl/k6tq2

Parra-Zhizhingo, Y., García-Herrera, D., Ávila-Mediavilla, C., \& Erazo-Álvarez, J. (2020). Plataformas Virtuales: retos y perspectivas a partir de Docentes [Virtual Platforms: challenges and perspectives from Teachers]. Revista Arbitrada Interdisciplinaria Koinonía, 5(5), 233-249. http://dx.doi.org/10.35381/r.k.v5i5.1041

Puma-Pañora, N., García-Herrera, D., Ochoa-Encalada, S., \& Erazo-Álvarez, J. (2020). Estrategias lúdicas para la vinculación familiar en el desarrollo infantil integral [Playful strategies for family bonding in comprehensive child development]. Revista Arbitrada Interdisciplinaria Koinonía, 5(5), 178-199. doi:http://dx.doi.org/10.35381/r.k.v5i5.1039

Sánchez, M. (2014). Epilepsia y deporte [Epilepsy and sport]. Universidad de Zaragosa. https://n9.cl/0te4z

Vancini, R. L., Andrade, M., Vancini-Campanharo, C. R., \& Lira, C. (2017). Exercise and sport do not trigger seizures in children and adolescents with epilepsy in school settings. Arquivos de neuro-psiquiatria, 75(10), 761. https://doi.org/10.1590/0004-282X20170111

Volpato, N. (2017). Level of physical activity and aerobic capacity. Plos One, 1-13. https://doi.org/10.1371/journal.pone.0181505 